\title{
Spike detection and sorting: combining algebraic differentiations with ICA
}

\author{
Zoran Tiganj $^{1}$ and Mamadou Mboup ${ }^{2,1}$ \\ 1 Projet ALIEN, INRIA Futurs \\ Parc Scientifique de la Haute Borne, \\ 40, avenue Halley Bt.A, Park Plaza, 59650 Villeneuve d'Ascq, Frace \\ 2 UFR Mathématiques et Informatique (CRIP5), \\ Université Paris Descartes, \\ 45 rue des Saints-Péres, 75270 Paris cedex 06, France
}

\begin{abstract}
A new method for action potentials detection is proposed. The method is based on a numerical differentiation, as recently introduced from operational calculus. We show that it has good performance as compared to existing methods. We also combine the proposed method with ICA in order to obtain spike sorting.
\end{abstract}

\section{Introduction}

Decoding the neural information is one of the most important problems in neuroscience. As it is well known, this information is conveyed by the spike train of electrical discharges, called action potential. Action potentials are generated when the equilibrium of electrical charges across the axonal membrane of a neuron is broken [1]. Decoding of the communication between neurons is an extremely challenging problem. One of the difficulties stems from the impossibility, in general, to record the activity of a single neuron but only a mixture activity of all neurons in a measured region. Imperative requirement for the neural information decoding is the ability of action potential detection and sorting (finding out which action potentials are fired by the same neuron) [2], [3], [4], [5].

To make the detection and sorting easier, recording systems usually consist of several electrodes. Each electrode receives the action potentials from all the surrounding neurons. The contribution from a single neuron depends on its distance from the electrode and on the type of the tissue that the action potentials go through. It is shown that ICA [6], [7] has a strong potential in neural signals detection and sorting [8], [9], even though it is well known that neurons are not independent sources - they are communicating with each other and the breaking of the equilibrium of electrical charges is caused by that communication.

In this paper we show how a new algebraic technique for numerical differentiation [10] can lead to a very good performances in neural spike detection. Further on, we use a combination of the technique with ICA to perform spike sorting.

In a neural recording settings, the number of neurons significantly exceeds that of the intracranial electrodes. We assume a measurement configuration 
where the distances between the neurons and the electrodes are such that the contribution of only few closest neurons will have a strong influence on the electrodes. The spiking activity of the remaining neurons is considered as the background noise [11].

The neural signal is first represented using a local piecewise polynomial model. The occurrence of an action potential (a spike) is represented by a discontinuity point in the model. Now, such singularities are fully characterized and easy to handle by the differential algebra and operational calculus approach to parameter estimation, introduced in [12] (see also [10] and [13]). Following this approach, we give in section 2 an explicit characterization of the spiking instants, as solutions of a polynomial equation, whose coefficients are composed of a short time window iterated integrals of the signal observation. Using classical numerical integration, we implement these coefficients as outputs of FIR filters. The filters are, as a matter of fact, numerical differentiators and moreover, they inherit the very important low pass property of the iterated integrals. The proposed method is compared in section 3 with two among the most successful methods in the literature. The simulation is performed using synthetic signal. We also combine our detection approach with ICA (see section 4). Benefit of such approach is not only a spike detection, but also a spike sorting, done by ICA. Some concluding remarks are pointed out in section 5 .

\section{Characterization of the spike instants}

In this section we give an explicit characterization of the spiking instants. More precisely we show that if an action potential occurs at time instant $t^{\star}$, then this may be described as a solution of a polynomial equation.

\subsection{Mathematical model}

To begin, the noisy observation spike train $y(t)$ is represented by the piecewise regular model

$$
y(t)=\sum_{i=1}^{K} \chi_{\left[t_{i-1}, t_{i}\right]}(t) p_{i}\left(t-t_{i-1}\right)+n(t),
$$

where $\chi_{\left[t_{i-1}, t_{i}\right]}(t)$ is the characteristic function of the interval $\left[t_{i-1}, t_{i}\right]$, the $t_{i}$ are the spiking instants and $n(t)$ is the noise corruption. In the sequel, we denote by $x(t)=y(t)-n(t)$ the unobserved noise-free signal. Each $p_{i}(t)$ is assumed to be a polynomial. Set $t_{0}=0$. Let $T$ be given such that there is at most one discontinuity point in each interval $I_{\tau}^{T}=(\tau-T, \tau), \tau \geq T$. Let set $x_{\tau}(t)=x(\tau-$ $t), t \in[0, T]$ for the restriction of the signal in $I_{\tau}^{T}$ and redefine the discontinuity point, say $t_{\tau}$, relatively to $I_{\tau}^{T}$ with:

$-t_{\tau}=0$ if $x_{\tau}(t)$ is smooth

$-0<t_{\tau} \leq T$ otherwise 
Now, the $N^{t h}$ order derivative of $x_{\tau}(t)$ (in the sense of distributions theory [14]) satisfies

$$
\frac{d^{N}}{d t^{N}} x_{\tau}(t)=\left[x_{\tau}^{(N)}\right](t)+\sum_{k=1}^{N}\left(\mu_{N-k}^{0} \delta(t)^{(k-1)}+\mu_{N-k} \delta\left(t-t_{\tau}\right)^{(k-1)}\right)
$$

where:

$-\mu_{k}^{0}=x^{(k)}(0+)-x^{(k)}(0-)=x^{(k)}(0)$

- $\mu_{k}$ is the jump of the $k^{t h}$ order derivative at the point $t_{\tau}$

$$
\mu_{k}=x^{(k)}\left(t_{\tau^{+}}\right)-x^{(k)}\left(t_{\tau^{-}}\right)
$$

with:

- $\mu_{0}=\mu_{1}=\ldots=\mu_{N-1}=0$ if there is no spike (action potential)

- $\mu_{k} \neq 0,0 \leq t_{\tau} \leq T$ if there is a spike (action potential)

where the notation $f^{(k)}$ means $k^{\text {th }}$ order derivative of $f$.

- $\left[x_{\tau}^{(N)}\right]$ represents the regular part of the $N^{t h}$ order derivative of the signal.

The action potential detection problem is now casted into the estimation of the location of the signal discontinuities, viz spike locations, $t_{\tau}$.

We assume that the degree of each $p_{i}(t)$ in (1) does not exceed $N-1$. The regular part $\left[x_{\tau}^{(N)}\right]$ then vanishes. Notice that different choices for $N$ lead to different descriptions of $t_{\tau}$. In the sequel we set $N=2$. Equation (2) then reduces to

$$
\frac{d^{2}}{d t^{2}} x_{\tau}(t)=\sum_{k=1}^{2}\left(\mu_{2-k}^{0} \delta(t)^{(k-1)}+\mu_{2-k} \delta\left(t-t_{\tau}\right)^{(k-1)}\right)
$$

\subsection{Finding the firing instants}

In order to solve the equation (3) for $t_{\tau}$, the problem is transferred into the operational domain (it is possible to solve the equation in the time domain too, but it is easier to handle expression like $\delta(t)$ using operational calculus) where equation (3) reads as:

$$
s^{2} \hat{x}_{\tau}(s)-s x_{\tau}(0)-\dot{x}_{\tau}(0)=\mu_{1} e^{-t_{\tau} s}+s \mu_{0} e^{-t_{\tau} s}
$$

Unknown parameters $\mu_{0}, \mu_{1}$ and initial conditions $x_{\tau}(0)$ and $\dot{x}_{\tau}(0)$ are irrelevant for our purpose. We consider them as undesired perturbations which are easy to eliminate by a $N^{t h}$ order differentiation with respect to $s$. This results in:

$$
t_{\tau}^{2}\left(s^{2} \hat{x}_{\tau}\right)^{(2)}+2 t_{\tau}\left(s^{2} \hat{x}_{\tau}\right)^{(3)}+\left(s^{2} \hat{x}_{\tau}\right)^{(4)}=0
$$

Multiplication by $s^{k}, k>0$ in the operational domain implies $k$ order derivative in the time domain. Now differentiation is known to be difficult and illconditioned. On the other hand division by $s^{\nu}, \nu>0$ corresponds in the time domain to the $\nu^{\text {th }}$ order iterated integral

$$
\frac{\hat{u}}{s^{\nu}} \rightarrow f(t)=\frac{1}{(\nu-1) !} \int_{0}^{t}(t-\lambda)^{\nu-1} u(\lambda) d \lambda .
$$


Let us then divide equation (5) by $s^{\nu}, \nu>2$. The resulting equation becomes in the time domain:

$$
\begin{aligned}
t_{\tau}^{2} \int_{0}^{t}(t-\lambda)^{\nu-1} \lambda^{2} x_{\tau}^{(2)}(\lambda) d \lambda-2 t_{\tau} \int_{0}^{t}(t-\lambda)^{\nu-1} \lambda^{3} x_{\tau}^{(2)}(\lambda) d \lambda \\
\quad+\int_{0}^{t}(t-\lambda)^{\nu-1} \lambda^{4} x_{\tau}^{(2)}(\lambda) d \lambda=0 .
\end{aligned}
$$

In the sequel we fix $t$ to $t=T$. Next, we integrate by parts and we replace the unobserved signal $x_{\tau}(t)$ by its noisy observation counterpart $y_{\tau}(t)=y(\tau-t)=$ $x(\tau-t)+n(\tau-t)$. This leads to the explicit characterization of $\hat{t_{\tau}}$ :

$$
\begin{array}{r}
{\hat{t_{\tau}}}^{2} \int_{0}^{T}\left[(T-\lambda)^{\nu-1} \lambda^{2}\right]^{(2)} y(\tau-\lambda) d \lambda-2 \hat{t_{\tau}} \int_{0}^{T}\left[(T-\lambda)^{\nu-1} \lambda^{3}\right]^{(2)} y(\tau-\lambda) d \lambda \\
+\int_{0}^{T}\left[(T-\lambda)^{\nu-1} \lambda^{4}\right]^{(2)} y(\tau-\lambda) d \lambda=0 \quad
\end{array}
$$

Let us define:

$$
h_{g}(\lambda)= \begin{cases}{\left[(T-\lambda)^{\nu-1} \lambda^{g+1}\right]^{(2)}} & 0 \leq \lambda<T \\ 0 & \text { otherwise }\end{cases}
$$

where $g=1,2,3$. After inserting $h_{g}(\lambda)$ back in equation (7) we obtain

$$
{\hat{t_{\tau}}}^{2} \int_{0}^{T} h_{1}(\lambda) y(\tau-\lambda) d \lambda-2 \hat{t_{\tau}} \int_{0}^{T} h_{2}(\lambda) y(\tau-\lambda) d \lambda+\int_{0}^{T} h_{3}(\lambda) y(\tau-\lambda) d \lambda=0
$$

The coefficients of this equation can be easily implemented as FIR filters.

Only discrete samples of the observation are available. We assume a regular sampling with the sampling period $T_{s}$. Let $\tau=n T_{s}$ and $T=M T_{s}$. Using a numerical integration method with abscissas $m T_{s}$ and weight $W_{m}, m=0, \ldots, M$, we obtain the discrete approximation of equation (9) given by

$$
{\hat{t_{\tau}}}^{2} \sum_{m=0}^{M} h_{1, m} y_{n-m}-2 \hat{t_{\tau}} \sum_{m=0}^{M} h_{2, m} y_{n-m}+\sum_{m=0}^{M} h_{3, m} y_{n-m}=0
$$

where: $h_{g, m}=W_{m} h_{g}\left(m T_{s}\right)$ and $y_{m}=y\left(m T_{s}\right)$. Trapezoidal rule for numerical integration defines the parameters $W_{m}$ :

$$
W_{0}=W_{M}=\frac{T_{s}}{2}, W_{i}=1, i=1,2, \ldots, M-1
$$

\section{Spike detection algorithm}

Recall that if the input observation $x_{\tau}$ is smooth, then all the three coefficients of the second order equation (6) are equal to 0 and we have $t_{\tau}=0$. Otherwise, 
the spiking instant satisfies $0 \leqslant t_{\tau} \leqslant T$. In the discrete time domain each of these coefficients can be derived as the input observation $x_{\tau}$ filtered with one of the three filters $\left\{h_{g, m}\right\}, g=1,2,3$ from equation (10). In the presence of a noise, $y_{\tau}=x_{\tau}+n_{\tau}$, each filter's output will be different from 0 , wether $x_{\tau}$ is smooth or not. But still, the values of the filters outputs will be highly correlated with the smoothness of the noise-free observation $x_{\tau}$ (see [10] where it is shown that the filters behave as a numerical differentiators). Therefore we use a product of the three filter's outputs as the final spike detection decision parameter. In this way, the occurrences of the spikes are highly emphasized with respect to the noise.

Comparison of the proposed detection method with two methods among the most accurate ones in the literature is hereafter presented. These are the method based on a combination of median filter, matched filter and nonlinear energy operator (NEO) [4] and the method based on wavelet transformation [5]. The spike train observation data are simulated ${ }^{1}$ as in [4]. The value of the parameter $T$, that defines the filter window length, is set to be $20 \%$ above the simulated spike length $(T=100$ samples). Figure 1 displays the receiver operating characteristic (ROC) curves for the different methods. These results show that the proposed
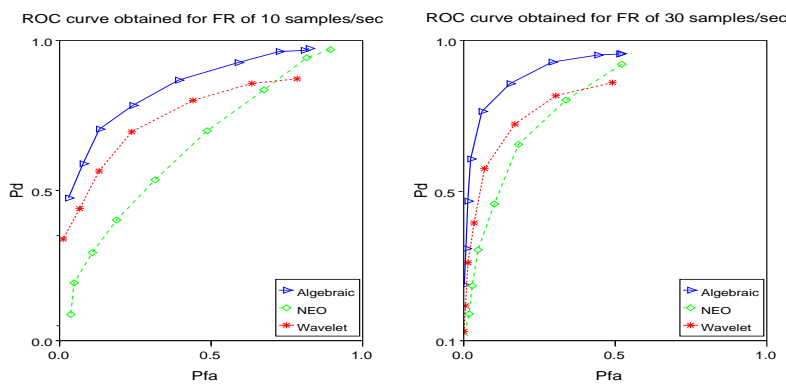

Fig. 1. ROC curves obtained by using the proposed method (Algebraic), NEO, and wavelet based method. Figures for two different firing rates (FR) are shown.

method compares favorably with respect to the others.

\section{Combination with ICA}

In this section, we use a more elaborated neural model for the simulation. This is described first. The description is followed by the implementation of the algebraic differentiation filters with ICA. Finally the simulation results are presented.

\footnotetext{
1 The signal is created using a code provided by Bruce Land at http://www.nbb. cornell. edu/neurobio/land/PROJECTS/spikeSort.
} 


\subsection{Creating the model}

Signals for the simulation are created from cell attached recording performed simultaneously on one of the Purkinje cells ${ }^{2}$ (figure 2(a)). Two seconds activity, with $15 \mathrm{kHz}$ sampling frequency (30000 samples in total), of 1000 artificial neurons are simulated. For this, spikes extracted from the recording were aligned into three clusters by using a classical $K$-means algorithm. Three different templates (figure 2(b)) are obtained by the centroids of each cluster.

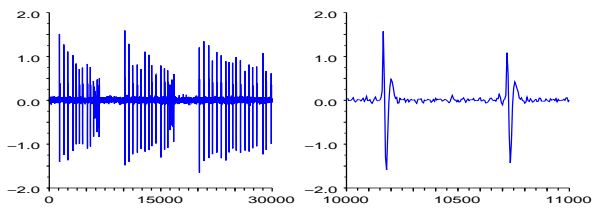

(a) Cell attached recording on one of Purkinje cells (left: 30000 recorded samples; right: zoom with 1000 samples)

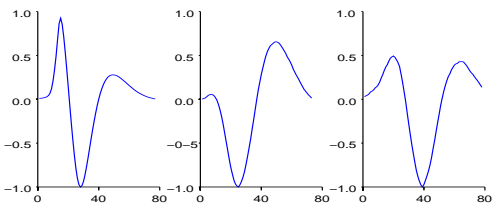

(b) Three action potential templates

Fig. 2. Cell attached recording and action potential templates

For each neuron, we simulate first the firing instants as Poisson distributed with a refractory period of $6.7 \mathrm{~ms}$. To each location, we then associate randomly one of the above spike templates. The neurons are spatially randomly distributed in the form of a cube, with minimal and maximal mutual distances. Four electrodes are placed on one side of the cube. We assume an isotropic medium (constant conductivity of the tissue), so that the contribution of each neuron is determined only by its distance from each electrode.

\subsection{Spike detection and sorting}

Superposition of the distant neurons activity occurs as a noise on the electrodes - original action potential shape waveforms are destroyed. Only the action potentials coming from the neurons that are close to the electrodes are recorded as spike shape waveforms. Next, signals from the four electrodes are applied to each of the three filters in order to emphasize action potential shape waveforms. The product of the filters outputs at each electrode may be used as a decision function. This was done in the previous section. Here we use the filters outputs as inputs for ICA in order to perform both, spike detection and sorting. We apply ICA on each set of the four outputs from each of the three filters. The goal is to reconstruct the activity of one neuron per electrode (the closest one).

\footnotetext{
${ }^{2}$ Signals are downloaded from C. Pouzat web page: http://www.biomedicale.univ-paris5.fr/physcerv/C_Pouzat/Data.html see [15] for more information
} 
FastICA MATLAB toolbox ${ }^{3}$ is used for ICA implementation. ICA gives three results for each of the three filters. The final detection and sorting result is given as a product of these three ICA outputs.

\section{$4.3 \quad$ Results}

To gauge the effects of the filters, we also consider the same settings, but with the filters replaced by the identity: we apply ICA directly on the signals from the electrodes and then raise each ICA output to the power 3 . Figure 3 compares the two normalized results obtained with and without the filters, on one sample realization of the reconstructed activity of one neuron. The figure shows that the amplitudes of the spikes that are not fired by the neuron closest to the observed electrode are attenuated more with the proposed method than when only ICA is used. This is confirmed in Table 1 that displays the ratio of the

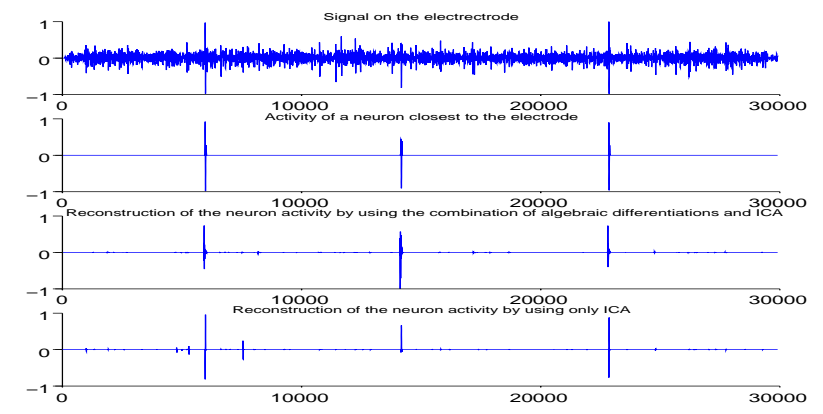

Fig. 3. Results of spike detection and sorting on one electrode with 30000 samples when only ICA is applied and when the combinations of algebraic differentiations and ICA is applied.

mean amplitude of the correctly detected action potentials (15 in total) over the mean amplitude of the falsely detected ones ( 80 in total). At a given electrode, a correct detection is meant when the detected action potential stems from the closest neuron. Otherwise, the detection is qualified as false. The first row of the table concerns the combination of the algebraic differentiations and ICA while the second row is for ICA alone. The different columns correspond to the four electrodes.

\section{Conclusion}

We have proposed a piecewise regular model for the spike train where the occurrence of a spike (transient) is represented by a discontinuity points. Using

\footnotetext{
3 The toolbox is developed at Laboratory of Information and Computer Science in the Helsinki University of Technology and it is available on http://www.cis.hut.fi/projects/ica/fastica/
} 
Table 1. Comparison of action potentials (AP) sorting when ICA is combined with the algebraic differentiation filters and when only ICA is used

\begin{tabular}{|c|l|l|l|l|}
\hline & \multicolumn{4}{|l|}{$\begin{array}{l}\text { Averaged normalized amplitudes of correctly detected AP / Av- } \\
\text { eraged normalized amplitudes of falsely detected AP }\end{array}$} \\
\hline & Neuron 1 & Neuron 2 & Neuron 3 & Neuron 4 \\
\hline AD and ICA & 29.45 & 48.97 & 29.76 & 86.31 \\
\hline ICA & 19.72 & 46.80 & 15.41 & 78.12 \\
\hline
\end{tabular}

operational calculus and basic algebraic manipulations we give an explicit characterization of these discontinuity points. The characterization provides a spike detection method which compares favorably with the existing methods. By combining this approach with ICA we obtain not only good results for detection, but also for spike sorting.

\section{References}

1. Rieke, F., Warland, D., de Ruyter van Steveninck, R., Bialek, W., Spikes: Exploring the neural code, MIT Press, 1999

2. Lewicki, M. S.: A review of methods for spike sorting: the detection and classification of neural action potentials, Network: Comput. Neural Syst., 1998

3. Delescluse, M., Pouzat, C.: Efficient spike-sorting of multi-state neurons using interspike intervals information, J Neurosci Methods, vol. 150, no. 1, pp. 16-29, 2006

4. Land, B., Spence, A.: Spike (action potential) detection and sorting, Journal Club 25, April 2002

5. Nenadic, Z., Burdick, J. W.: Spike detection using the continious wavelet transform, IEEE Trans. Biomed. Eng., vol. 52, no. 1, pp. 74-87, 2005.

6. Cardoso, J.F.: Blind signal separation: statistical principles, Proceedings of the IEEE, vol. 10, no. 9, p.p. 2009-2025, 1998

7. Comon, P.: Independent component analysis, A new concept?, Sig. Proc. 36, 1994

8. Mamlouka, A. M., Sharp, H., Menne, K. M.L., Hofmann, U. G., Martinetz, T.: Unsupervised spike sorting with ICA and its evaluation using GENESIS simulations, Neurocomputing, 2005

9. Brown, G. D., Yamada, S., Sejnowski, T., J.: Independent component analysis at the neural cocktail party, TRENDS in Neurosciences, 2001

10. Mboup, M., Join, C., Fliess, M. Numerical differentiation with annihilators in noisy environment, to appear in: Numerical Algorithms, 2008.

11. Gerstner, W., Kistler W.: Spiking Neuron Models. Single neurons, Populations, Plasticity, Cambridge University Press, 2002

12. Fliess, M., Sira-Ramirez, H.: An algebraic framework for linear identification ESAIM Contr. Opt. Calc. Variat., VOL. 9, 2003.

13. Mboup, M.: Parameter estimation for signals described by differential equations, To appear in Applicable Analysis, 2008. DOI: 10.1080/00036810802555441

14. Schwartz, L.: Theorie des distributions, ed. 3, Hermann, 1998

15. Pouzat, C.: Methods and Models in Neurophysics. Les Houches 2003 Summer School, pp. 729-786, Elsevier, 2005, Available from: http://fr.arxiv.org/abs/q-bio.QM/0405012 